\title{
Acronyms Used in This Book
}

[The following list, although not comprehensive, includes important acronyms that appear frequently in the chapters that follow. Unfortunately, it was not always possible to discover the precise meanings of acronyms referring to longdefunct political groups and other organizations.-Ed.]

AFLS Agence Française de Lutte contre le Sida

AMG Association des Médecins Gais

ARCL Archives Lesbiennes

$\mathrm{BEH} \quad$ Bulletin Epidémologique Hebdomadaire

CADAC Coordination Nationale des Associations pour le Droit à l'Avortement et à la Contraception

CAPR Comité d'Action Pédérastique Révolutionnaire

CERM Centre d'Etudes et de Recherches Marxistes

CFES Comité Français d'Education pour la Santé

CGL Centre Gai et Lesbien

CGT Confédération Générale du Travail

CNIL Commission National Informatique et Libertés

CNTS Centre National de Transfusion Sanguine

CUARH Comité d'Urgence Anti-Répression Homosexuelle

CUC Contrat d'Union Civile

CUS Contrat d'Union Sociale

CVS Contrat de Vie Sociale

DGS Direction Générale de la Santé

EHESS Ecole des Hautes Etudes en Sciences Sociales

FHAR Front Homosexuel d'Action Révolutionnaire

FLJ Front de Libération des Jeunes

FLN Front de Libération Nationale

FMA Féminin, Masculin, Avenir

GLH Groupes de Libération Homosexuelle

GP Gauche Prolétarienne

JCR Jeunesse Communiste Révolutionnaire

LCR Ligue Communiste Révolutionnaire

LO Lutte Ouvrière 


\author{
LSD Lesbiennes Se Déchaînent \\ MDC Mouvement des Citoyens \\ MIEL Mouvement d'Information et d'Expression des Lesbiennes \\ MLAC Mouvement de la Libération de l'Avortement et de la Contraception \\ MLF Mouvement de la Libération des Femmes \\ OCT Organisation Communiste des Travailleurs \\ PCF Parti Communiste Français \\ PSU Parti Socialiste Unifié \\ SID Service d'Information et de Diffusion \\ SIS Sida Infos Service \\ SNEG Syndicat National des Entreprises Gaies \\ UEC Union d'Etudiants Communistes \\ UNADIF Union Nationale des Associations de Déportés \\ UNEF Union Nationale des Etudiants Français \\ VLR Vive la Révolution
}

